\title{
Tyrosinase-mediated Peptide Conjugation with Chitosan-coated Gold Nanoparticles
}

\author{
Naomi Sakono,* Kosuke Nakamura,* Tatsuki OhShima, $* *$ Ryoto Hayakawa, $* *$ and \\ Masafumi SAKONO**;
}

*Department of Applied Chemistry and Chemical Engineering, National Institute of Technology, Toyama College, 13 Hongo, Toyama, Toyama 939-8630, Japan

**Department of Applied Chemistry, Faculty of Engineering, University of Toyama, 3190 Gofuku, Toyama, Toyama 930-8555, Japan

\begin{abstract}
The conjugation of biomolecules, such as protein, sugar, and DNA, with metal nanoparticles is an important technique for bioassay and biomaterial preparation. In this study, we aim to enzymatically immobilize a functional peptide on gold nanoparticles (AuNPs) using a single-step reaction. We used tyrosinase, a catechol oxidase, to immobilize an enzymatic peptide. We performed immobilization experiments of a fluorescent compound-linked caspase-3 substrate peptide using tyrosinase on chitosan-coated AuNPs. Peptides were effectively immobilized onto the AuNPs depending on the presence of tyrosine within the sequence, which suggests the DOPA-quinone produced from tyrosine, via tyrosinase, is connected to the chitosan amino group. Although fluorescent emission from the immobilized capase- 3 substrate was quenched by AuNPs, fluorescence intensity recovery occurred due to the addition of caspase-3. Thus, we were able to easily prepare functional AuNPs that can be used for a caspase-3 activity assay. Our results indicate that the tyrosinase-mediated peptide link to chitosan-coated particles is a useful technique for preparing functionalized nanoparticles.
\end{abstract}

Keywords Bioconjugation, caspase-3 activity assay, functional nanoparticles, tyrosinase

(Received September 12, 2018; Accepted November 8, 2018; Advance Publication Released Online by J-STAGE November 16, 2018)

\section{Introduction}

Conjugating gold nanoparticles (AuNPs) with biomacromolecules is a widely used technique for various biomaterials and bioassays. ${ }^{1,2}$ AuNPs exhibit characteristic optoelectronic properties based on their size and shape. ${ }^{3}$ Gold nanoparticles have strong absorption from 500 to $550 \mathrm{~nm}$ because of surface plasmon resonance (SPR). ${ }^{4,5}$ Since the SPR band absorption peak is sensitive to the surrounding environment, AuNPs are suitable for colorimetric sensing. ${ }^{6,7}$ Moreover, AuNPs are considered to be excellent biotechnology tools because they have unique features, such as a large surface-to-volume ratio, excellent biocompatibility, and low toxicity. ${ }^{8}$

Generally, AuNP preparation involves tetrachloroaurate(III) chemical reduction using agents such as citric acid and sodium borohydride in an aqueous medium. ${ }^{9,10}$ Since colloidal suspensions tend to aggregate easily due to thermodynamic instability, controlling AuNP aggregation is highly important. Major approaches to prevent aggregation include using protective agents, which offset Van der Waals attractive forces via steric and electrostatic repulsion. Thus, AuNPs are prepared using chemical reduction techniques with protective agents such as poly(vinylpyrrolidone), ${ }^{11-13}$ polyethylene glycol, ${ }^{14,15}$ and chitosan. ${ }^{16,17}$ For biotechnological applications, AuNPs require conjugation with biomolecules. Principal methods inducing

† To whom correspondence should be addressed.

E-mail: msakono@eng.u-toyama.ac.jp biomolecule immobilization onto an AuNP's surface include ionic or hydrophobic adsorption interactions and thiol for metal surface binding. ${ }^{18}$ Although conjugation via adsorption is simple, adsorbed biomolecule inactivation occurs easily due to structural denaturation and inconsistent orientation. The interaction between thiol and the metal surface solves the adsorption problems. However, optimizing the immobilization efficiency is necessary due to reaction competition between disulfide bond formation and thiol-metal binding. ${ }^{19}$ Hence, these difficulties mean more desirable and effective nanoparticle bioconjugation methods need to be developed.

In this study, we examined enzyme-mediated peptide immobilization with chitosan-coated AuNPs. We used tyrosinase, a catechol oxidase, for immobilization. Recently, oxidative enzymes, such as tyrosinases, ${ }^{20,21}$ and peroxidases, ${ }^{22}$ have been frequently used to generate chitosan conjugates. Tyrosinase is widely distributed in nature; it causes the skins of fruits and vegetables to ripen or become brown and catalytically converts phenols into $o$-quinones. ${ }^{23}$ DOPA-quinone, converted from tyrosine via tyrosinase, indicates nucleophile reactivity towards the amine and sulfhydryl groups. ${ }^{24}$ Due to DOPAquinone's high reactivity with amino groups contained within chitosan, tyrosinase is used to prepare chitosan-protein conjugation. ${ }^{25}$ Hence, we suggest that functional peptides can be conjugated with chitosan, which functions as a protective agent during AuNP synthesis via tyrosinase (Scheme 1). As a model, we prepared AuNPs immobilized with peptides that include the caspase- 3 substrate sequence. We performed the caspase- 3 activity assay using the prepared nanoparticles. 


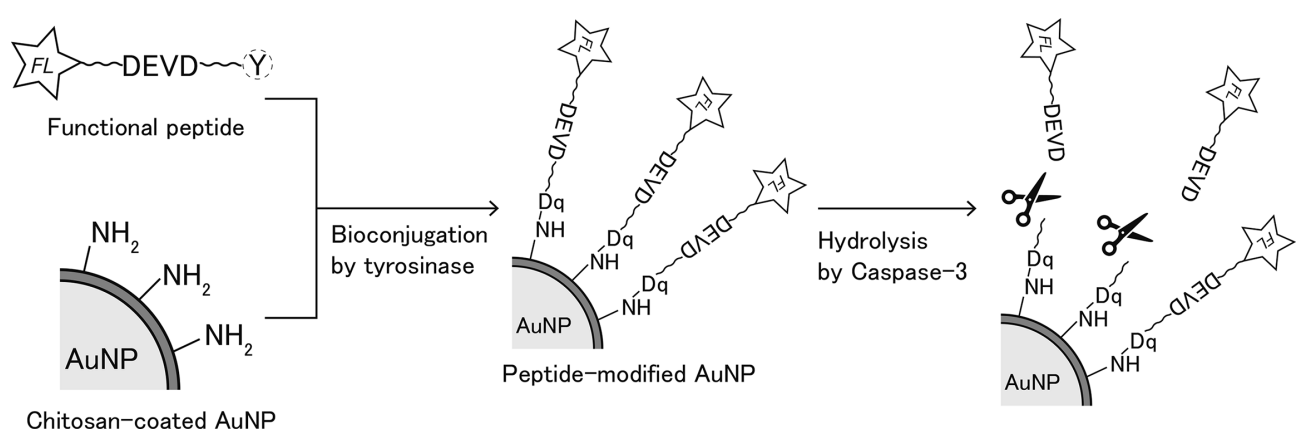

Scheme 1 A schematic illustration of AuNP enzymatic preparation, which functions as a caspase-3 substrate. The terms Dq and FL represent the DOPA-quinone and fluorescent compound, respectively.

\section{Experimental}

\section{Materials}

Hydrogen tetrachloroaurate(III) tetrahydrate, chitosan-10, and general reagents were purchased from Wako Pure Chemical Industries (Osaka, Japan). Tyrosinase and sodium borohydride were obtained from Sigma-Aldrich (St. Louis, MO, USA). Acetyl-Asp-Glu-Val-Asp-H (Ac-DEVD-CHO) was purchased from Peptide Institute (Osaka, Japan). Functional peptides bound with fluorescent compounds (P1: FAM-GGGSDEVDGGGSY-COOH, P2: FAM-GGGSDEVDGGGS-COOH) were obtained from the peptide synthesis service provided by Eurofins Genomics Japan (Tokyo, Japan).

\section{Preparation and characterization of chitosan-coated AuNP}

Chitosan $(0.9 \mathrm{wt} \%)$ was fully dissolved in a $1 \mathrm{wt} \%$ acetic acid solution. Ten millimolar $\mathrm{HAuCl}_{4}$ was added to the chitosansolved solution and stirred for $15 \mathrm{~min}$ at $25^{\circ} \mathrm{C}$. Subsequently, $40 \mathrm{mM} \mathrm{NaBH}_{4}$ was added gently to the solution and stirred for 30 min. Prepared AuNPs were collected via centrifugation at $6000 \mathrm{rpm}$ for $150 \mathrm{~min}$ (Himac CT15E, Hitachi). The supernatant was removed via micropipette, after which the collected AuNPs were resuspended in pure water or phosphate buffered saline (PBS). For microscopic observation, AuNP-containing solutions were placed on carbon-coated copper grids and air-dried. The samples were analyzed using an electron microscope $(\mathrm{H}-7650$, Hitachi). The histogram of size distribution was built up by counting more than 130 particles. We also confirmed AuNP yield using UV-Vis spectroscopy (UV-2450, Shimadzu corporation). A concentration of the produced AuNPs (absorbance 1.0 at $520 \mathrm{~nm}$ ) was estimated to be approximately $22.2 \mathrm{nM}$ based on average diameter (about $6.5 \mathrm{~nm}$ ) calculated from a histogram. ${ }^{26}$

\section{FT-IR measurements}

FT-IR chitosan-coated AuNP spectra were recorded using FT-IR spectroscopic techniques (FT/IR-4200, JASCO). After lyophilization of chitosan-coated AuNPs suspended in water, measurement samples were mixed with $\mathrm{KBr}$. We used the Kaleida Graph (Hulinks) software to apply the smoothing processes to the recorded spectra.

\section{FAM fluorescence measurements}

Functional peptides $(10 \mu \mathrm{M})$ solubilized in PBS were moved to quartz cells with a $1.0-\mathrm{cm}$ optical path length. Fluorescent spectra excited at $490 \mathrm{~nm}$ were recorded from 500 to $800 \mathrm{~nm}$ using the LS-55 spectrofluorometer (Perkin Elmer) at $25^{\circ} \mathrm{C}$.
The peptide-immobilized AuNPs suspended in PBS were placed in a 96 well black microplate, after which fluorescent intensity was measured using a microplate reader (Appliskan, Thermo Fisher Scientific) equipped with a 480-nm excitation and $535 \mathrm{~nm}$ emission filter.

\section{Functional peptide immobilization using tyrosinase}

Functional peptide $(10 \mu \mathrm{M})$ and chitosan-coated AuNPs were mixed with $100 \mathrm{nM}$ tyrosinase in a PBS solution. We adjusted the amount of AuNPs to 1.0 at an absorbance of $520 \mathrm{~nm}$. The reaction mixture was incubated for $2 \mathrm{~h}$ at $37^{\circ} \mathrm{C}$. AuNPs were collected via centrifugation at $6000 \mathrm{rpm}$ for $1 \mathrm{~h}$ after incubation and washed with PBS. We confirmed peptide immobilization using a fluorescence assay with the microplate reader described previously.

\section{Caspase-3-mediated hydrolysis reaction}

The peptide-modified AuNPs (absorbance 1.0 at $520 \mathrm{~nm}$ ), suspended in PBS, were collected via centrifugation at $6000 \mathrm{rpm}$ for $1 \mathrm{~h}$. The supernatant was removed via micropipette. Ten microlitres of PBS, containing a certain unit of caspase- 3 in the presence or absence of $10 \mu \mathrm{M}$ Ac-DEVD-CHO which works as caspase- 3 inhibitor, were added to the collection tube, and the AuNPs were well dispersed by pipetting. The mixture was incubated for $1 \mathrm{~h}$ at $37^{\circ} \mathrm{C}$. After incubation, $200 \mu \mathrm{L}$ PBS was added to the collection tube. The fluorescence assay, using the microplate reader, was performed without removing the AuNPs from the sample.

\section{Results and Discussion}

First, we assessed AuNP properties when prepared in the presence of chitosan. The UV-Vis spectrum for AuNPs suspended in pure water is characterized by typical absorbance features arising from SPR (Fig. 1(A)) as well as an intense band located at approximately $520 \mathrm{~nm}$. The prepared AuNP morphology was analyzed using transmission electron microscopy (TEM) (Fig. 1(B)). Observations from TEM suggest that AuNPs with round shapes are distributed in groups based on small size, i.e., less than approximately $10 \mathrm{~nm}$. Figure 1(C) indicates AuNP size distribution based on TEM observations. Particles that have diameters between 5 and $6 \mathrm{~nm}$ are produced mainly by $\mathrm{HAuCl}_{4}$ reduction in the presence of chitosan. Next, we confirmed whether chitosan exists with the AuNPs. We measured the FT-IR spectra for the lyophilized AuNP samples after cleaning them with water. Based on the spectra, typical amine $\left(\mathrm{NH}_{2}\right)$ bands were observed near 3400 


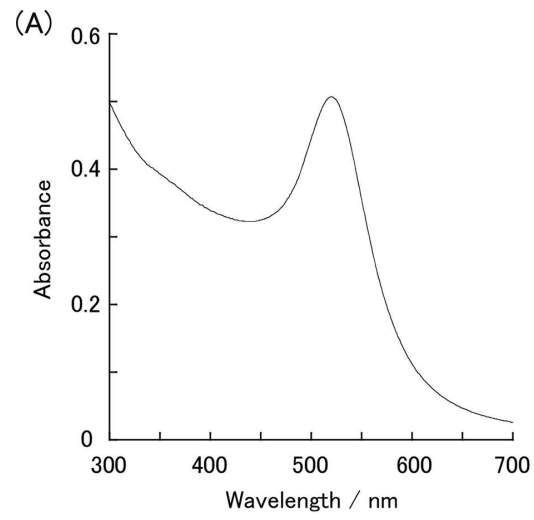

(C)

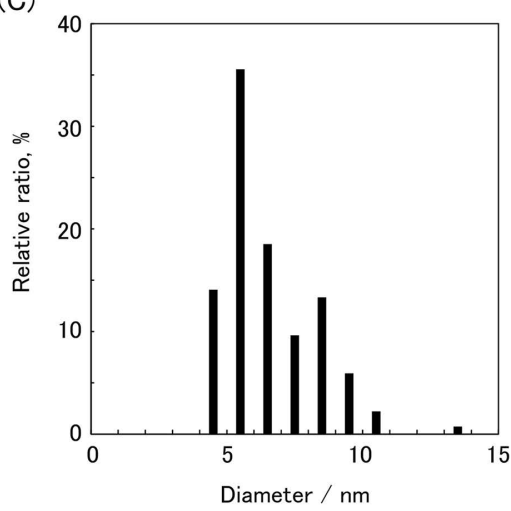

(B)

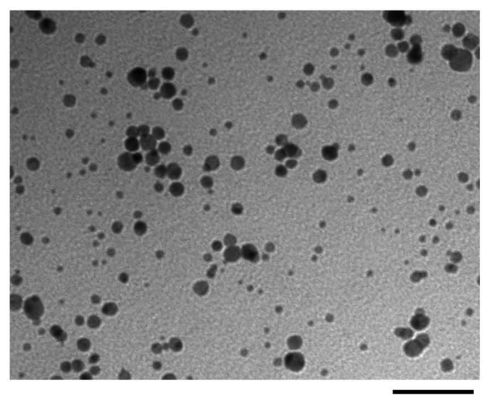

(D)

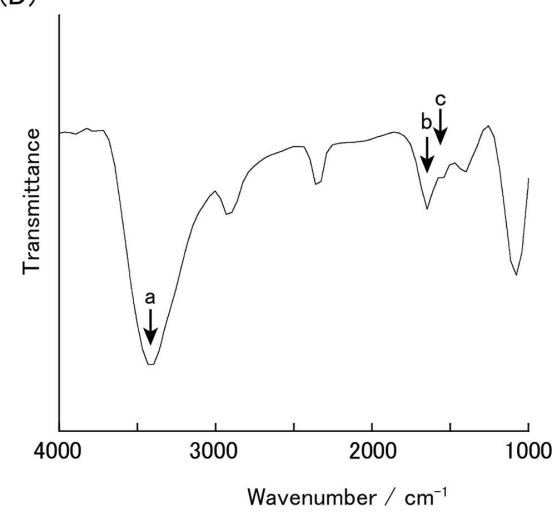

Fig. 1 Prepared AuNP physical properties. (A) UV-Vis spectroscopy. (B) TEM observations. Scale bar indicates $50 \mathrm{~nm}$. (C) Diameter distribution histogram. (D) FT-IR spectrum. Arrows a, b, and c indicate 3400,1590 , and $1560 \mathrm{~cm}^{-1}$, respectively.

and $1590 \mathrm{~cm}^{-1}$. We also observed a carbonyl $(\mathrm{C}=\mathrm{O})$ band near $1560 \mathrm{~cm}^{-1}$. Since these bands are characteristic of chitosan, ${ }^{27,28}$ the AuNP FT-IR spectra strongly suggest that the newly produced AuNPs complexed with chitosan. In general, AuNPs form aggregates under conditions that have high salt concentrations. The newly produced AuNPs' stability was confirmed against a buffer solution. We measured the UV-Vis spectra for samples incubated for $24 \mathrm{~h}$ after replacement with PBS solvent. As shown in Fig. 2, spectra for AuNPs suspended in PBS were similar to those for AuNPs suspended in water. This result indicates that newly produced AuNPs in chitosan are stable in the buffer solution, which implies that chitosan, coated onto the AuNP surface, prevented interaction between particles.

Different peptide sequences, i.e., P1 and P2, were immobilized onto the chitosan-coated AuNPs where there was a tyrosine presence at the $\mathrm{C}$-terminal. Fluorescent properties for these peptides were measured using fluorescence spectroscopy. As shown in Fig. 3, each peptide is characterized by an emission peak near $520 \mathrm{~nm}$ with excitation at $480 \mathrm{~nm}$. Obvious differences for spectra shapes were not confirmed, suggesting that tyrosine at the C-terminal does not influence fluorescent properties.

Subsequently, we performed enzymatic functional peptide immobilization using tyrosinase on chitosan-coated AuNPs. After immobilization reactions, supernatant fluorescence, from the centrifuged reaction mixture, was measured to assess the unbound peptides. Figure 4 shows peptide fluorescent intensity within the supernatant from the reaction mixture. The P1 peptide is significantly influenced by the supernatant's fluorescence intensity when exposed to tyrosinase. On the other

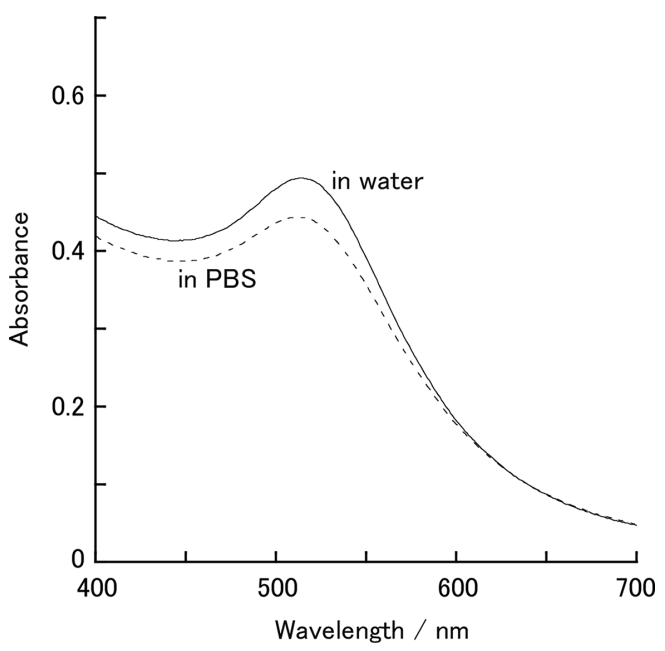

Fig. 2 Absorption spectra for AuNPs suspended in water or PBS incubated for $24 \mathrm{~h}$ after buffer exchange.

hand, we did not observe a clear difference for the P2 peptide. This result indicates that the $\mathrm{P} 1$ peptide was effectively immobilized on chitosan-coated AuNPs unlike the P2 peptide. Thus, we suggest that peptide immobilization depends on the amount of tyrosine within the P1 peptide. From the results discussed above, we successfully prepared functional peptidemodified AuNPs using tyrosinase.

The P1 peptide contains an amino acid sequence DEVD, 
which is the substrate for caspase- $3 .^{29}$ The caspase- 3 catalyzes the hydrolysis reaction with the DEVD-containing peptide, yielding a peptide divided into two fragments. Therefore, in the presence of caspase-3, we expect part of the P1 peptide that is immobilized on AuNPs is removed from the particle via hydrolysis (Scheme 1). Caspase-3 activity can be estimated via the fluorescent dye's fluorescence intensity in the detached peptide. After incubation for the caspase- 3 hydrolysis reaction, the reaction mixture's fluorescent intensity was measured using the microplate reader without separating the AuNPs from the mixture. Figure 5(A) shows the relationship between fluorescent intensity and the amount of caspase-3. We observed an increase in the fluorescent intensity that depends on the increasing of caspase-3. Moreover, the regaining of fluorescence in the presence of caspase-3 inhibitor was markedly decreased. These results indicate that the $\mathrm{P} 1$ peptide, which was immobilized on the AuNPs, functions as a caspase- 3 substrate. Increases in the fluorescent intensity were observed despite an unseparated reaction mixture. This result presumes that particle-immobilized, FAM fluorescence emissions were quenched by the AuNPs. Gold and silver nanoparticles function as strong fluorescent quenchers that are localized near nanoparticle surfaces..$^{30,31}$ Thus, we speculate that fluorescent mechanisms increase via

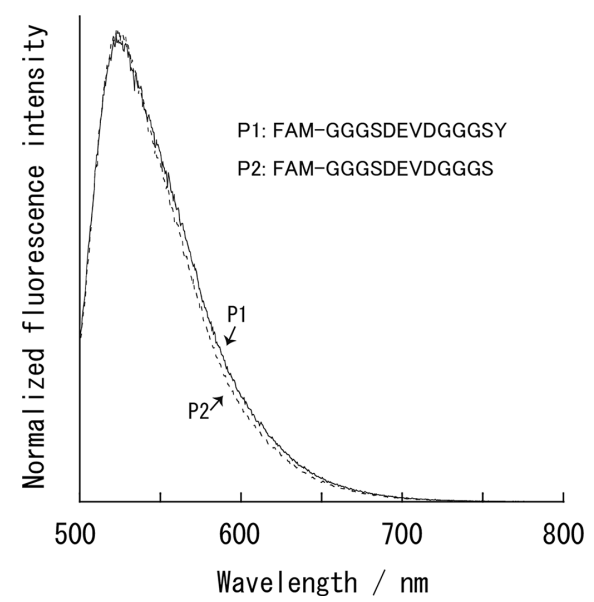

Fig. 3 Fluorescent spectra for the functional peptides. Fluorescent spectra excited at $490 \mathrm{~nm}$ were recorded from 500 to $800 \mathrm{~nm}$ using a spectrofluorometer. hydrolysis reactions, as shown in Fig. 5(B). As particleimmobilized FAM emission is quenched by the AuNPs, fluorescent intensity is less than one, which is estimated from the amount of immobilized functional peptide. The fluorescent chemical is detached from the AuNP surface when caspase- 3 is mixed with peptide-modified AuNPs. As a result, there is recovery of the quenched fluorescence intensity. Hence, increases in fluorescent intensity reflect caspase- 3 activity.

\section{Conclusions}

We immobilized the peptide on AuNPs enzymatically. Chitosan, used in this study, has two roles: 1) it is used as an aggregation inhibitor among nanoparticles, chitosan-coated nanoparticles were characterized by stable conditions in PBS solutions for long periods, and 2) it acts as a scaffold to immobilize peptides via a link between DOPA-quinone and the amino group within the chitosan. By using the tyrosinase-mediated link, caspase-3 substrate immobilization onto AuNPs was performed
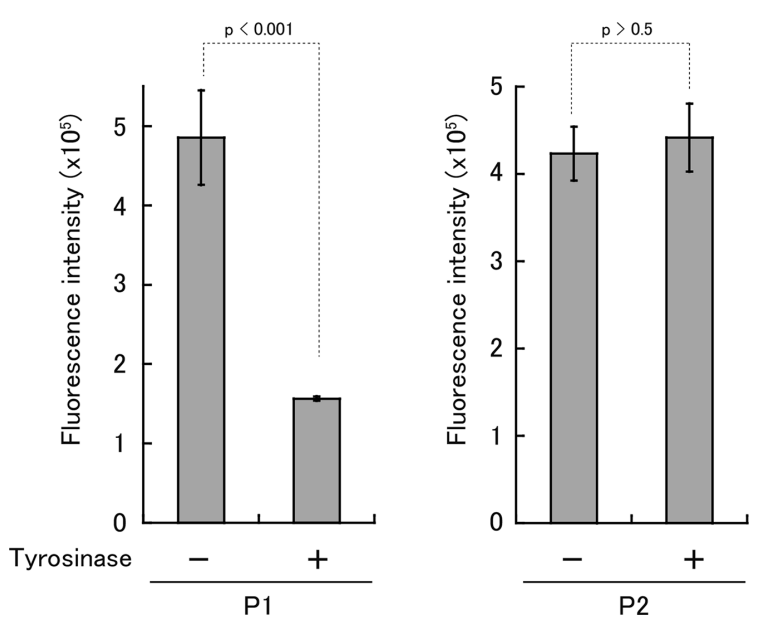

Fig. 4 Supernatant fluorescence intensity in mixture after the immobilization reaction. Symbol + and - indicate with and without tyrosinase in the immobilization reaction, respectively. Fluorescence intensity was measured using a microplate reader equipped with a 480-nm excitation and a 535-nm emission filter. The values shown are the mean $\pm \mathrm{SE}$ ( standard error; $n=3$ ).
(A)

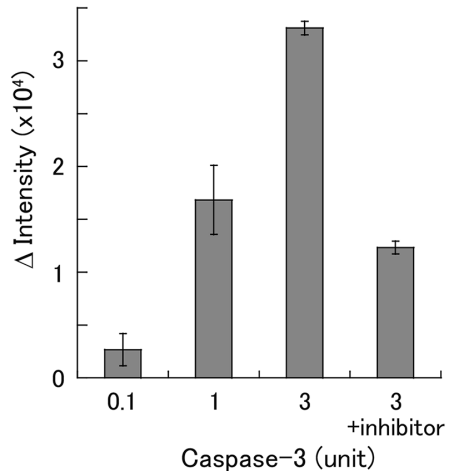

(B)

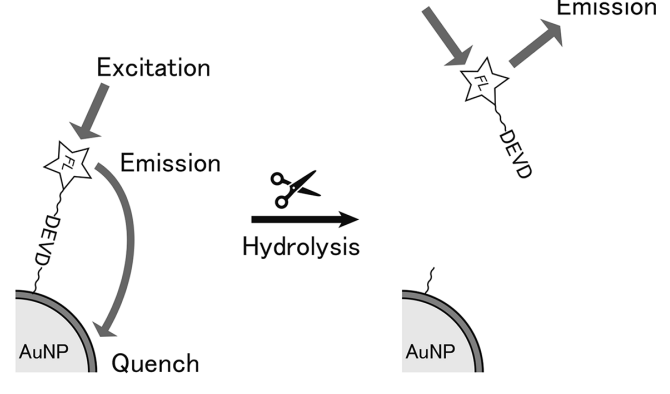

Fig. 5 Hydrolysis reactions via caspase-3. (A) Fluorescence intensity differences after the addition of caspase- 3 in the presence or absence of caspase- 3 inhibitor. The values shown are the mean $\pm \mathrm{SE}$ (standard error; $n=3$ ). (B) Estimated mechanisms for increasing fluorescence intensity via hydrolysis. 
successfully. Although FAM fluorescent emission linked with the caspase- 3 substrate was quenched due to a transfer of energy to the AuNPs, increased fluorescent intensity was observed by adding caspase-3. Thus, peptide-modified AuNPs are applicable to caspase- 3 activity assay. As caspases are apoptosis-related factors, the nanoparticles produced by our method are expected to contribute to biochemical assays involving cell death.

A metal nanoparticle functionalization via peptide and protein modification is an important technique, especially for a colorimetric assay. Since a tyrosinase-mediated link occurs between tyrosine and amino groups, a site-specific modification is possible by accounting for the peptide sequence or amino acid that appears on the protein.

\section{Acknowledgements}

We thank the Toyama University Research and Development in Natural Science Center for TEM analysis. This work was supported by a Grant-in-Aid for Young Scientists (B) (15K17445) from Japan Society for the Promotion of Science (to N. S.).

\section{References}

1. M. De, P. S. Ghosh, and V. M. Rotello, Adv. Mater., 2008, 20, 4225 .

2. N. Elahi, M. Kamali, and M. H. Baghersad, Talanta, 2018, 184, 537.

3. T. K. Sau, A. L. Rogach, F. Jäckel, T. A. Klar, and J. Feldmann, Adv. Mater, 2010, 22, 1805.

4. K. L. Kelly, E. Coronado, L. L. Zhao, and G. C. Schatz, J. Phys. Chem. B, 2003, 107, 668.

5. S. Eustis and M. A. El-Sayed, Chem. Soc. Rev., 2006, 35, 209.

6. N. Sui, F. Liu, T. Li, L. Wang, T. Wang, M. Liu, and W. W. Yu, Anal. Sci., 2017, 33, 963.

7. Y. Kong, J. Shen, and A. Fan, Anal. Sci., 2017, 33, 925.

8. N. Khlebtsov and L. Dykman, Chem. Soc. Rev., 2011, 40, 1647.

9. K. R. Brown, D. G. Walter, and M. J. Natan, Chem. Mater. 2000, 12, 306.

10. J. Turkevich, P. C. Stevenson, and J. Hillier, Discuss. Faraday Soc., 1951, 11, 55.

11. M. H. Ullah, T. Hossain, and C. S. Ha, J. Mater. Sci., 2011,
46,6988 .

12. T. Teranishi, I. Kiyokawa, and M. Miyake, Adv. Mater. 1998, 10, 596.

13. N. Hashimoto, N. Nishida, H. Murayama, and H. Tanaka, Chem. Lett., 2011, 40, 144.

14. P. Free, C. P. Shaw, and R. Lévy, Chem. Commun., 2009, 5009.

15. L. F. Leopold, I. S. Tódor, Z. Diaconeasa, D. Rugină, A. Ştefancu, N. Leopold, and C. Coman, Colloids Surf., A, 2017, 532, 70 .

16. H. Huang and X. Yang, Biomacromolecules, 2004, 5, 2340.

17. Z. Chen, Z. Wang, X. Chen, H. Xu, and J. Liu, J. Nanoparticle Res., 2013, 15, 1930.

18. M. H. Jazayeri, H. Amani, A. A. Pourfatollah, H. PazokiToroudi, and B. Sedighimoghaddam, Sens. Bio-Sensing Res., 2016, 9, 17.

19. F. Li, H. Zhang, B. Dever, X.-F. Li, and X. C. Le, Bioconjugate Chem., 2013, 24, 1790.

20. A. Demolliens, C. Boucher, Y. Durocher, M. Jolicoeur, M. D. Buschmann, and G. De Crescenzo, Bioconjugate Chem., 2008, 19, 1849.

21. A. Anghileri, R. Lantto, K. Kruus, C. Arosio, and G. Freddi, J. Biotechnol., 2007, 127, 508.

22. L. Vachoud, T. Chen, G. F. Payne, and R. Vazquez-Duhalt, Enzyme Microb. Technol., 2001, 29, 380.

23. M. R. Loizzo, R. Tundis, and F. Menichini, Compr. Rev. Food Sci. Food Saf., 2012, 11, 378.

24. J. Buchert, D. Ercili Cura, H. Ma, C. Gasparetti, E. Monogioudi, G. Faccio, M. Mattinen, H. Boer, R. Partanen, E. Selinheimo, R. Lantto, and K. Kruus, Annu. Rev. Food Sci. Technol., 2010, 1, 113.

25. T. Chen, R. Vazquez-Duhalt, C. F. Wu, W. E. Bentley, and G. F. Payne, Biomacromolecules, 2001, 2, 456.

26. W. Haiss, N. T. K. Thanh, J. Aveyard, and D. G. Fernig, Anal. Chem., 2007, 79, 4215.

27. Z. Osman and A. K. Arof, Electrochim. Acta, 2003, 48, 993.

28. Z. Li, Y. Du, Z. Zhang, and D. Pang, React. Funct. Polym., 2003, 55, 35 .

29. M. Poreba, A. Strózyk, G. S. Salvesen, and M. Drag, Cold Spring Harbor Perspect. Biol., 2013, doi: 10.1101/ cshperspect.a008680.

30. Y. Tu, P. Wu, H. Zhang, and C. Cai, Chem. Commun., 2012, 48, 10718.

31. E. Dulkeith, M. Ringler, T. A. Klar, J. Feldmann, A. M. Javier, and W. J. Parak, Nano Lett., 2005, 5, 585. 